• 研究报告・

\title{
施氮水平影响蝶蚓介导的番茄生长及抗虫性
}

\author{
张 宇 ${ }^{1}$ 肖正高 1 蒋林惠 ${ }^{1}$ 钱 蕾 2 陈小云 ${ }^{1}$ \\ 陈法军 2 胡 锋 ${ }^{1}$ 刘满强 ${ }^{*}$
}

1 (南京农业大学资源与环境科学学院土壤生态实验室, 南京 210095)

2 (南京农业大学植物保护学院, 南京 210095)

\begin{abstract}
摘要: 过量施用氮肥不仅导致严重的生态环境问题，还会限制土壤生物驱动的生态系统服务功能。蚳蚂的取食和 掘穴等活动可以促进土壤肥力和植物生长, 进而影响植物与病虫害的关系。了解氮肥与害虫作用下蚚蚂对植物抗 虫性的影响, 有助于揭示土壤动物的生态功能调控机制。采用蚯蚂(威廉腔环蚓Metaphire guillelmi)、西花蓟马 (Frankliniella occidentalis)和氮肥用量的三因子完全交互设计, 利用番茄(Lycopersicon esculentum)盆栽实验探索了 不同氮水平下蚯蚓对番茄生长及对植食者抗性的影响。结果表明, 在低氮条件下蝶蚂显著降低了番茄茎叶干生物 量、根系干生物量及茎叶可溶性糖含量, 而茎叶的茉莉酸和水杨酸含量分别是无蝶蚓对照的 6 倍和 3 倍, 且伴随 着西花薊马数量下降了 $58 \%$ 。在高氮水平时, 蚯蚓未影响番茄茎叶茉莉酸、茎叶水杨酸含量及西花薊马数量。蚟 蚂介导的番茄营养物质(茎叶可溶性糖和茎叶全氮)和防御信号物质(茎叶茉莉酸和茎叶水杨酸)含量分别与西花薊 马数量呈显著的正相关和负相关。总之, 氮肥施用改变的土壤氮有效性通过改变植物资源和防御物质含量转变了 蛏蚂介导的植物抗虫性响应; 全面了解土壤生物对植物生长的影响需要综合考虑土壤管理-土壤动物-植物病原 物三者之间的关系。
\end{abstract}

关键词: 土壤动物; 植物化学组成; 害虫; 地上和地下部; 生态系统服务

\section{Nitrogen levels modify earthworm-mediated tomato growth and resistance to pests}

Yu Zhang ${ }^{1}$, Zhenggao Xiao ${ }^{1}$, Linhui Jiang ${ }^{1}$, Lei Qian², Xiaoyun Chen ${ }^{1}$, Fajun $\mathrm{Chen}^{2}$, Feng $\mathrm{Hu}^{1}$, Manqiang $\mathrm{Liu}^{1 *}$

1 Soil Ecology Laboratory, College of Resources and Environmental Sciences, Nanjing Agricultural University, Nanjing 210095

2 College of Plant Protection, Nanjing Agricultural University, Nanjing 210095

\begin{abstract}
Excessive chemical nitrogen $(\mathrm{N})$ fertilizer application causes serious environmental problems and affects the ecosystem services that depend on soil biota. Earthworms improve soil fertility and plant productivity via activities such as feeding, burrowing and casting, and alter the relationships between crop plants and pests by modifying plant primary and secondary productivity. In order to mechanistically understand the functional roles of soil fauna in ecosystem services, a pot experiment using tomatoes (Lycopersicon esculentum) was conducted in a greenhouse. The study used a complete factorial design that manipulated earthworm (Metaphire guillelmi) abundance, western flower thrips (Frankliniella occidentalis) and $\mathrm{N}$ input. Results showed that under low $\mathrm{N}$-input conditions, earthworms significantly reduced shoot and root biomass and shoot soluble sugar content, and increased shoot jasmonic acid content (by 6 times) and shoot salicylic acid content (by 3 times), compared to treatments without earthworms. This was accompanied by the decrease of thrips abundance by $58 \%$, indicating a remarkable suppression of aboveground pests by earthworms. However, under high $\mathrm{N}$-input conditions, earthworm presence did not affect the contents of shoot jasmonic acid or shoot salicylic acid or thrips abundance by the end of the experimental period (45 days). The earthworm-mediated responses of plant nutrition (shoot soluble sugar and shoot total nitrogen)
\end{abstract}


was significantly positively correlated with thrips abundance, whereas defense (shoot jasmonic and salicylic acid) was significantly negatively correlated. Changes to soil $\mathrm{N}$ availability due to $\mathrm{N}$ fertilizer input can shift the direction of earthworm-mediated plant resistance against herbivores by altering plant resource acquisition and secondary defense. Effects of earthworms on plant growth and resistance depend on soil management practices such as $\mathrm{N}$ fertilizer application. A comprehensive understanding of the roles of soil biota in mediating plant growth requires knowledge of the multifaceted relationships among soil management, soil fauna, and plant pathogens.

Key words: soil fauna; plant chemistry; pests; aboveground-belowground; ecosystem service

随着人口增长引起的农产品需求增加, 高强度 集约化管理正成为促进农业生产的重要手段 (Bommarco et al, 2013; 徐炜等, 2016)。然而, 高强 度土地利用方式会导致土壤生物多样性丧失和生 态系统服务功能受损(Tsiafouli et al, 2015; 潘开文 等, 2016)。此外, 过量施用化肥也是降低作物抗病 虫能力及导致病虫害暴发的主要因素(Larbat et al, 2012); 化学肥料施用引发的农药依赖性增强的反 馈作用会进一步破坏生态平衡, 使人类的生产活动 及人类健康与生态环境走向恶性循环(Aurélien et al, 2013; Bender et al, 2016)。在保护生态环境和农业可 持续发展的要求下, 挖掘土壤生物的有益功能, 加 强对植物一土壤生物之间互作关系的认识, 以减少 对化肥农药的依赖, 指导病虫害综合管理和促进生 态农业发展。因此, 系统了解关键土壤生物的生态 功能及其对植物生长和抗虫性的影响机制, 显得尤 为迫切且重要。

自然界的植物在生长过程中往往遭受植食者 的取食压力, 在与植食者协同进化的过程中, 植物 会进化出各种策略以提高自身的防御能力(Kempel et al, 2011)。土壤施肥措施特别是氮肥施用引起的 土壤养分变化又调控着植物对植食者的抵抗能力 (Bogaert et al, 2017)。氮素直接参与植物体内的生理 生化过程并改变植物体内的组织液成分, 影响植物 的营养组成(氨基酸和糖类等), 最终影响植食者的 取食和植物的防御响应, 如土壤的高氮水平会使得 植物体含氮量高、提高植物对害虫的适口性(Staley et al, 2010); 而氮素贵乏会导致植物因缺乏营养而 不能正常生长甚至降低自身抗性引发害虫取食 (Shekoofa \& Emam, 2008)。越来越多的研究表明, 土壤生物对地上部系统从个体到群落及功能水平 上均起着调控作用(傅声雷, 2007; 王邵军和阮宏华, 2008; Korres et al, 2016)。土壤生物能够直接或间接 调控根系这一枢纽进而对植物地上部性状产生影
响(吴纪华等, 2007; Bardgett \& van der Putten, 2014; Schädler \& Ballhorn, 2017)。例如, 研究发现菌根真 菌、根际促生菌等能促进植物的生长并调控植物抵 御病虫害的能力(Aurélien et al, 2013; Tao et al, 2016)。已有的蚯蚓方面的研究表明, 蝶蚓可以促进 植物对养分的吸收(Zhang et al, 2010), 其对植物促 生效应的大小与方向还取决于土壤氮素水平(van Groenigen et al, 2014)。虽然蝶蚓对植物抗虫性的 影响也取决于氮素水平, 但是却缺乏足够的实验 证据。

一般认为提高土壤氮素有效性是蚚蚓促进植 物生长或生产力的最主要机制 (Bertrand et al, 2015)。荟萃分析归纳出蝶蚓影响植物生长的效果在 土壤氮素贫痊时更明显(van Groenigen et al, 2014)。 值得注意的是, 蚯蚓提高植物氮含量的同时也导致 以其为宿主的病虫害的发展(Wurst et al, 2010a)。氮 素水平还能够改变植物用于防御的次生代谢物质 的合成(Wurst et al, 2004), 间接影响植食者的取食 及行为(Poveda et al, 2005; Loranger-Merciris et al, 2012)。植物次生代谢物质总酚、信号分子茉莉酸和 水杨酸可以诱导一系列抗逆信号传导和防御反应, 可以抵御虫害(Caarls et al, 2015)。研究者发现, 蝶蚓 可提高植物生物量及养分含量, 增加植物耐受植食 者的取食胁迫(Blouin et al, 2005)。最近的荟萃分析 也表明了蚳蚓介导的植物抗性的资源分配模式受 到植食者类型及土壤养分等因素的影响(Xiao et al, 2018)。迄今, 有关蝶蚓对植食者影响的研究多集中 在刺吸者蚜虫上, 蛏蚓可以刺激抗性防御物质的产 生, 从而降低蚜虫的数量(Lohmann et al, 2009)。有 关虹蚓对其他类型害虫影响的研究仍缺乏，限制了 对虹蚓一植物关系调控作用的全面认识。虹蚓影响 植物生长和防御抗性的效应大小和方向会因各种 生物和环境条件而变(Wurst, 2013)。依据生长-抗性 权衡假说, 我们推测蝶蚓介导的植物生长与抗性之 
间的关系会依赖于土壤氮素水平, 氮素水平高低导 致的植物能量储备差异使植物生长和抗性关系发 生变化(Züst \& Agrawal, 2017)。例如, 当土壤氮素贵 乏时, 蚯蚓可促进矿化过程来缓解植物养分限制, 相应地, 植物储备能量的增加也提高了合成抗性物 质的能力; 当氮素充足时, 植物生长速率较快, 相 应合成的抗性物质含量较低, 在此条件下高植食压 力发挥主导作用, 而高氮施用带来的蚯蚓摄取食物 的元素不均衡及土壤结构和性质的改变, 可能会造 成蚯蚓生理胁迫, 使虾蚓对植物生长和抗性的影响 均较弱(Brown et al, 2004; Mundim et al, 2017; Zheng et al, 2018)。

西花蓟马(Frankliniella occidentalis)是全球最 重要的检疫性害虫之一, 严重危害蔬菜作物, 它以 锉吸式口器取食植物的茎叶及花果, 严重时导致植 物枯萎死亡(吕要斌等, 2011), 其对植物的破坏作用 并不亚于在蝶蚂研究中普遍关注的蚜虫。特别是考 虑到植食者与植物的相互关系在不同取食类型的 植食者间存在明显差异(Xiao et al, 2018), 因此有必 要了解更多植食者种类下蚯蚓的作用机制和影响因 素。基于比较施氮量对虾蚓介导的番茄生长及其对 西花蓟马抗性的影响, 探究蚟蚓介导的植物生长及 防御作用的影响，旨在系统地了解虾蚓的生态服务 功能及植物抗虫性调控机制, 指导农业的绿色发展。

\section{材料与方法}

\section{1 土壤的采集}

土壤采自江苏省如东县农业生态功能调控研 究基地的多年蔬菜地 $\left(120^{\circ} 42^{\prime} \mathrm{E}, 32^{\circ} 12^{\prime} \mathrm{N}\right)$, 属亚热 带季风气候, 年平均气温 $15^{\circ} \mathrm{C}$, 年均降水量 1,042 $\mathrm{mm}$ 。供试土壤为粘壤土, 取样深度为 $0-20 \mathrm{~cm}$ 。鲜 土采集后过 $5 \mathrm{~mm}$ 篮, 剔除大型土壤动物及根茬等 残体。土壤质地为 $6.48 \%$ 黏粒, 63.55\%粉粒, $29.97 \%$ 砂粒, 土壤 $\mathrm{pH}$ 值(水土比2.5: 1) 为7.75, 土壤硝态氮 含量为 $22.32 \mathrm{mg} / \mathrm{kg}$, 铵态氮含量为 $0.96 \mathrm{mg} / \mathrm{kg}$, 总 有机碳和全氮含量分别为 $10.73 \mathrm{~g} / \mathrm{kg}$ 和 $0.52 \mathrm{~g} / \mathrm{kg}$ 。

\section{2 实验设计和布置}

采用3因子, 即蚳蚓、西花蓟马(western flower thrips, WFT)和氮肥用量的交互实验设计, 设置不 施氮(0 kg N/ha) vs. 施高氮(240 kg N/ha), 获得土壤 低氮(Low N)和高氮( High $\mathrm{N}$ )两个水平, 施高氮处理 的施肥量为 $240 \mathrm{~kg} \mathrm{~N} / \mathrm{ha}$ 的尿素, 地上部植食者
$(-\mathrm{WFT}=$ 无西花蓟马,$+\mathrm{WFT}=$ 接种西花蓟马 $) 、$ 蚯 蚓 $(\mathrm{E} 0=$ 无虾蚓, $\mathrm{E} 1=$ 接入威廉腔环蚓(Metaphire guillelmi)) 3 个因子，重复6次，总共 $2 \times 2 \times 2 \times 6=$ 48盆。

供试植物为番茄 (Lycopersicon esculentum), 品 种为 “世纪红冠”，具有抗病性及耐热性好、高抗番 茄花叶病毒叶霉病、枯萎病和灰霉病等特性。挑选 饱满番茄种子，用 $10 \%$ 的 $\mathrm{H}_{2} \mathrm{O}_{2}$ 浸泡 30 min进行表面 灭菌并清洗，在无菌水中浸泡 $12 \mathrm{~h}$, 然后将番茄种 子置于湿纱布中 $25^{\circ} \mathrm{C}$ 下培养 $3 \mathrm{~d}$ 发芽, 长至幼苗待 用。供试西花蓟马由山东省农业科学院植物保护研 究所提供。

盆栽实验在人工气候室中进行(温度 $25 \pm 9 \mathrm{C}$, 光照时间 $16 \mathrm{~h}$, 光照强度 $15,000 \mathrm{lux})$ 。每盆装 $1.5 \mathrm{~kg}$ 干重当量的土壤，调节含水量为饱和持水量的 $60 \%$, 并在 $25^{\circ} \mathrm{C}$ 黑暗条件下培养 1 周。供试虾蚓为从深层 种向内层种的过渡类群, 现习惯称为内层种, 蚯蚂 采集与盆栽土壤的采集地点相同。选择大小及生物 量相近的虹蚓清洗表面, $48 \mathrm{~h}$ 排空肠道后洗净并称 重(Dalby et al, 1996)。基于虾蚓采集地田间密度(105 条 $\left./ \mathrm{m}^{2}\right)$, 虾蚓的接种量为每盆 3 条总共 $10 \pm 0.43 \mathrm{~g}$, 各处理具体接种量见表1。当番茄幼苗有3片完全叶 时移栽入盆, 所有盆钭每周随机换位3次以最小化 位置效应, 每 $2 \mathrm{~d}$ 浇 $50 \mathrm{~mL}$ 蒸馏水。待番茄生长 $30 \mathrm{~d}$ 时接入西花蓟马, 用软毛刷刷取成虫 20 头至 $2 \mathrm{~mL}$ 离 心管内待用, 雌雄比为 $1: 1$ 。每株番茄选择两片靠 近顶端的叶片进行接种，即每盆接种 40 头蓟马，用 250 目纱网罩封住已接虫叶片，防止西花蓟马逃逸。

\section{3 采样和保存}

西花蓟马接种 $15 \mathrm{~d}$ 后进行破坏性采样。整个盆 栽实验周期持续 $45 \mathrm{~d}$, 采样时先将西花蓟马从接虫 叶片上用吸虫管收集并计数。番茄植株地上部茎叶 和地下部的根系及土壤等分别采集。采样时番茄根 系几乎分布于整个土体。主要测定指标包括西花蓟 马的数量、番茄营养物质、番茄防御物质及信号物 质等。

\section{4 分析方法}

植株全氮采用 $\mathrm{H}_{2} \mathrm{SO}_{4}-\mathrm{H}_{2} \mathrm{O}_{2}$ 法消解, 凯氏定氮 法测定(鲁如坤, 2000)。植株激素用高效液相色谱串联质谱法(Hettenhausen et al, 2013)测定: 称取样品 液氮研磨, 加入交联聚乙烯吡咯烷酮(PVPP)和乙酸 乙酯冰浴超声后离心, 转移上清液到新离心管, 氮 
表 1 不同处理下威廉腔环蚓的数量及生物量变化

Table 1 Changes in biomass and number of Metaphire guillelmi under different treatments

\begin{tabular}{|c|c|c|c|c|c|c|}
\hline \multirow{2}{*}{$\begin{array}{l}\text { 处理 } \\
\text { Treatments }\end{array}$} & & \multicolumn{2}{|c|}{ 接种时 Inoculation time } & \multicolumn{2}{|c|}{ 采样时 Sampling time } & \multirow{2}{*}{$\begin{array}{l}\text { 蚯蚓失重率 } \\
\text { Weight loss of the } \\
\text { earthworm (\%) }\end{array}$} \\
\hline & & 数量 Number & 生物量 Biomass (g) & 数量 Number & 生物量 Biomass (g) & \\
\hline \multirow{2}{*}{$\begin{array}{l}\text { 无西花蓟马 } \\
\text {-WFT }\end{array}$} & \multirow{2}{*}{$\begin{array}{l}\text { 低氮 Low N } \\
\text { 高氮 High N }\end{array}$} & 3 & $10.49 \pm 0.27$ & 3 & $9.19 \pm 0.34$ & 12.39 \\
\hline & & 3 & $10.35 \pm 0.24$ & 3 & $8.63 \pm 0.47$ & 16.62 \\
\hline \multirow{2}{*}{$\begin{array}{l}\text { 接种西花蓟马 } \\
+ \text { +WFT }\end{array}$} & \multirow{2}{*}{$\begin{array}{l}\text { 低氮 Low N } \\
\text { 高氮 High N }\end{array}$} & 3 & $10.19 \pm 0.19$ & 3 & $8.52 \pm 0.22$ & 16.39 \\
\hline & & 3 & $10.27 \pm 0.26$ & 3 & $7.85 \pm 0.66$ & 23.56 \\
\hline
\end{tabular}

Low N, 0 kg N/ha urea; High N, 240 kg N/ha urea.

吹浓缩, 加入 $70 \%$ 甲醇浴旋后离心, 将上清液过 $0.22 \mu \mathrm{m}$ 滤膜, 保存待测。植株酚类的测定采用福林 酚比色方法(Ainsworth \& Gillespie, 2007): 取20 mg 植株研磨成粉, 加 $95 \%$ 甲醇后黑暗浸提。离心后取 上清液到新离心管, 添加斐林试剂和 $\mathrm{Na}_{2} \mathrm{CO}_{3}$ 后室 温黑暗反应 $2 \mathrm{~h}$, 读取其在波长 $765 \mathrm{~nm}$ 处的吸光值。

土壤全氮以半微量凯氏法测定。土壤微生物生 物量碳(MBC) 和土壤微生物生物量氮( $(M B N)$, 采用 氯仿熏蒸法测定。土壤可溶性有机碳(DOC), 称取 新鲜土壤于浸提瓶中加入超纯水振荡, 离心过滤膜 稀释后用 TOC 仪(Elementar, Germany)测定。利用连 续流动分析仪(SKALAR San++, Holland)测定土壤 铵态氮和硝态氮含量。

\section{5 数据分析}

运用R 3.3.2软件进行数据分析与作图, 利用 Kolmogorov-Smirnov和Levene方法检验数据的正态 分布及方差异质性。采用多因素方差分析估计蛏 蚓、氮素以及植食者因素对番茄生长和植物化学组 成等指标的影响。通过Pearson's相关性计算防御物 质茎叶茉莉酸、茎叶水杨酸与西花蓟马数量之间的 关系。

\section{结果}

\section{1 土壤性质变化}

虾蚓对土壤矿质氮、微生物生物量的影响取决 于氮素水平和西花蓟马(表2)。低氮时蝶蚓对土壤硝 态氮含量无影响, 高氮时降低了硝态氮含量(图1)。 在没有西花蓟马时, 虾蚓增加了铵态氮含量, 接种 西花蓟马后, 蚯蚂反而降低了铵态氮含量。接种蚯 蚓增加了土壤微生物生物量氮, 但降低了微生物生 物量碳含量(图1)。

\section{2 番茄生长及营养物质变化}

虹蚓对番茄生物量及营养物质的影响与氮素 水平和西花蓟马有关 $(P<0.05$, 表3)。蝐蚓和氮素的 交互作用显著影响根系生物量和茎叶可溶性糖含 量, 低氮时虹蚓降低根系生物量和茎叶可溶性糖, 而高氮下无影响(表3，图2)。蚯蚓作用增加了番茄茎 叶全氮含量, 但降低了根系全氮含量(图2)。

\section{3 番茄防御物质含量和西花蓟马数量的变化}

虾蚓对番茄茎叶茉莉酸、茎叶水杨酸及总酚的 影响与氮素水平及西花蓟马有关 $(P<0.05$, 表3)。有 西花蓟马时, 低氮下番茄的茎叶茉莉酸和水杨酸含

表 2 蚯蚓、氮素及植食者因素对土壤硝态氮、铵态氮、微生物生物量碳含量和微生物生物量氮含量影响的方差分析结果 Table 2 ANOVA results showing the effects of earthworm, nitrogen and herbivore on the contents of $\mathrm{NO}_{3}{ }^{-}-\mathrm{N}, \mathrm{NH}_{4}{ }^{+}-\mathrm{N}, \mathrm{microbial}^{-}$ biomass carbon and microbial biomass nitrogen in soils

\begin{tabular}{|c|c|c|c|c|c|c|c|}
\hline 变量 Variables & $\begin{array}{l}\text { 虾蚓 Earthworm } \\
\text { (E) }(d f=1)\end{array}$ & $\begin{array}{l}\text { 氮素 Nitrogen } \\
(\mathrm{N})(d f=1)\end{array}$ & $\begin{array}{l}\text { 西花蓟马 Thrips } \\
(\text { WFT) }(d f=1)\end{array}$ & $\begin{array}{l}\mathrm{E} \times \mathrm{N} \\
(d f=1)\end{array}$ & $\begin{array}{l}E \times W F T \\
(d f=1)\end{array}$ & $\begin{array}{l}\mathrm{N} \times \mathrm{WFT} \\
(d f=1)\end{array}$ & $\begin{array}{l}\mathrm{E} \times \mathrm{N} \times \mathrm{WFT} \\
(d f=1)\end{array}$ \\
\hline 硝态氮 $\mathrm{NO}_{3}^{-}-\mathrm{N}$ & $18.49^{* * *}$ & $87.43^{* * *}$ & $8.21^{* *}$ & $18.02^{* * * *}$ & 3.51 & $6.67 *$ & 3.68 \\
\hline 铵态氮 $\mathrm{NH}_{4}^{+}-\mathrm{N}$ & 4.07 & 2.06 & $10.82^{* *}$ & 1.91 & $27.26^{* * *}$ & 0.00 & 0.28 \\
\hline $\begin{array}{l}\text { 微生物生物量碳 } \\
\text { Microbial biomass carbon }\end{array}$ & $10.35^{* *}$ & 1.00 & $60.26^{* * *}$ & 2.66 & 0.99 & $15.51^{* * *}$ & 2.00 \\
\hline $\begin{array}{l}\text { 微生物生物量氮 } \\
\text { Microbial biomass nitrogen }\end{array}$ & $11.94^{* *}$ & 0.38 & 2.69 & 0.84 & 3.90 & 2.44 & $23.70^{* * *}$ \\
\hline
\end{tabular}

$* P<0.05, * * P<0.01, * * * P<0.001$. 
无虾蚓 Without earthworm
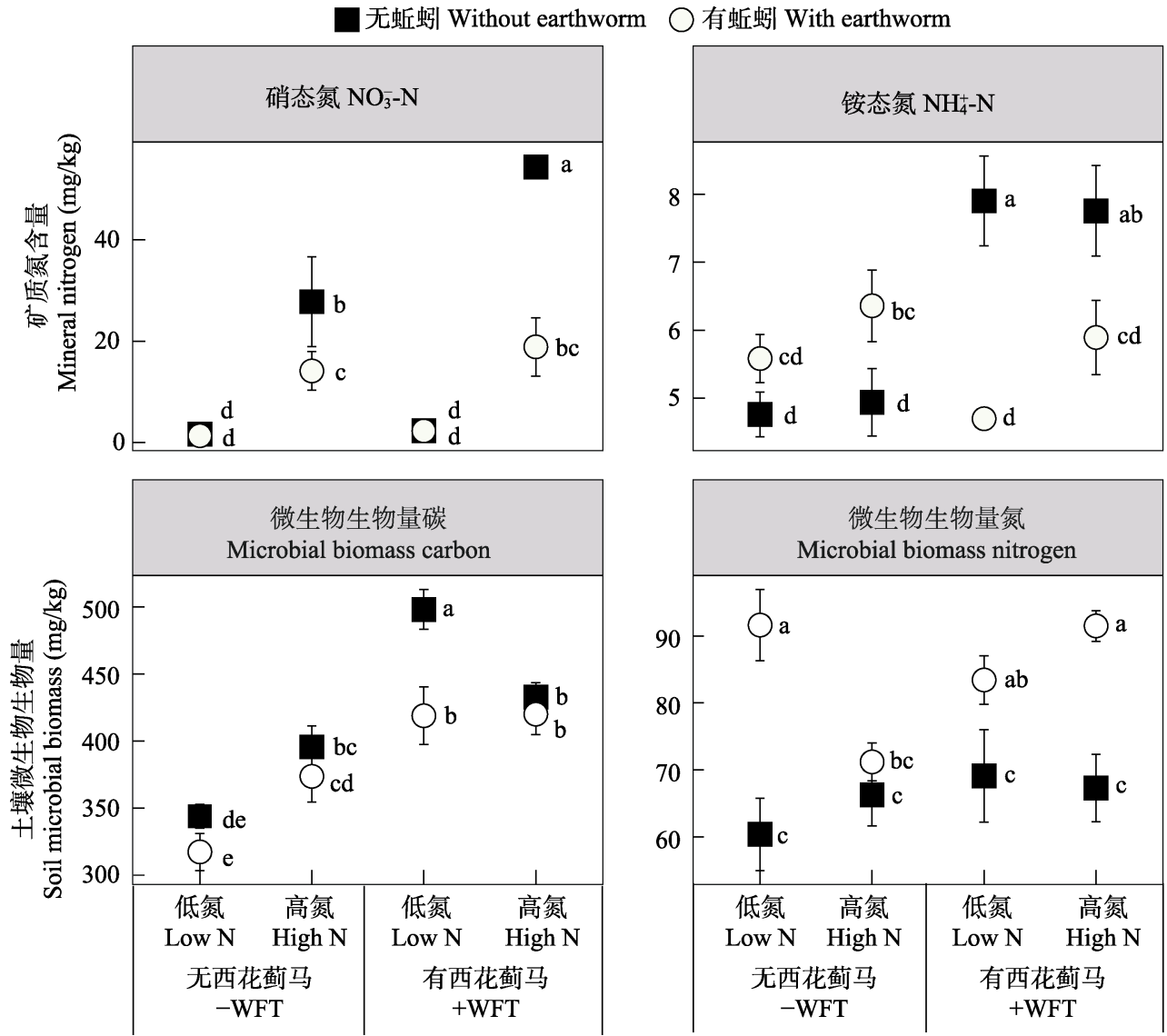

图 1 不同氮素条件下, 蚯蚓在西花蓟马(WFT)有无时对土壤硝态氮、铵态氮、微生物生物量碳和微生物生物量氮含量(平均 值 \pm 标准差, $n=6)$ 的影响。 $-W F T$ 和+WFT分别表示无西花蓟马和有西花蓟马。不同小写字母表示各处理间差异显著 (Fisher's LSD检验, $\boldsymbol{P}<\mathbf{0 . 0 5}$ )。

Fig. 1 Effects of earthworm Metaphire guillelmi on the contents of soil $\mathrm{NO}_{3}{ }^{-}-\mathrm{N}, \mathrm{NH}_{4}{ }^{+}-\mathrm{N}$, microbial biomass carbon and microbial biomass nitrogen in the absence or presence of western flower thrips (WFT) under low and high N inputs (mean \pm SD, $n=6$ ). In the figure, -WFT and +WFT indicate without and with western flower thrips, respectively. Means with different letters indicate significant difference among treatments (Fisher's LSD test, $P<0.05$ ). Error bars are standard errors.

\section{表 3 蚯蚓、氮素、植食者因素及其交互作用对番茄茎叶及根系营养和化学防御性状的影响}

Table 3 Effects of earthworm, nitrogen, herbivore and their interactions on the nutrients and chemical traits of tomato shoots and roots

\begin{tabular}{|c|c|c|c|c|c|c|c|}
\hline $\begin{array}{l}\text { 变量 } \\
\text { Variables }\end{array}$ & $\begin{array}{l}\text { 蚳蚓 Earthworm } \\
(\mathrm{E})(d f=1)\end{array}$ & $\begin{array}{l}\text { 氮素 Nitrogen } \\
(\mathrm{N})(d f=1)\end{array}$ & $\begin{array}{l}\text { 西花蓟马 Thrips } \\
(\text { WFT })(d f=1)\end{array}$ & $\begin{array}{l}E \times N \\
(d f=1)\end{array}$ & $\begin{array}{l}E \times W F T \\
(d f=1)\end{array}$ & $\begin{array}{l}\mathrm{N} \times \mathrm{WFT} \\
(d f=1)\end{array}$ & $\begin{array}{l}E \times N \times W F T \\
(d f=1)\end{array}$ \\
\hline 茎叶干生物量 Shoot dry biomass & $22.49^{* * *}$ & $117.88^{* * *}$ & $10.36^{* *}$ & 2.39 & 1.64 & $5.86^{*}$ & 0.05 \\
\hline 根系干生物量 Root dry biomass & $13.77^{* * *}$ & 2.08 & 0.20 & $11.47^{* *}$ & 0.00 & 0.22 & 1.68 \\
\hline 茎叶可溶性糖 Shoot soluble sugar & $25.21^{* * * *}$ & 2.09 & $15.47^{* * * *}$ & $17.79^{* * * *}$ & 2.01 & 0.03 & 0.03 \\
\hline 根系可溶性糖 Root soluble sugar & 0.01 & 0.71 & 2.68 & 2.17 & 3.69 & 1.39 & $13.09^{* * *}$ \\
\hline 茎叶全氮 Shoot total nitrogen & $12.21^{* *}$ & $182.83^{* * *}$ & 1.19 & 3.10 & 0.00 & 0.07 & 1.70 \\
\hline 根系全氮 Root total nitrogen & $20.37^{* * *}$ & $246.77^{* * *}$ & $15.21^{* * *}$ & 0.06 & $11.12^{* *}$ & 2.79 & 2.11 \\
\hline 茎叶游离氨基酸 Shoot amino acid & 0.97 & $20.43^{* * *}$ & $7.57^{* *}$ & 0.73 & 0.28 & $26.10^{* * *}$ & $8.31^{* *}$ \\
\hline 根系游离氨基酸 Root amino acid & $46.10^{* * *}$ & $43.08^{* * *}$ & $7.38^{* *}$ & $21.88^{* * *}$ & $14.80^{* * *}$ & $32.87^{* * *}$ & 0.96 \\
\hline 茎叶酚 Shoot phenolics & 2.36 & $36.23^{* * * *}$ & $4.75^{*}$ & 1.50 & 2.26 & 0.31 & $29.01^{* * *}$ \\
\hline 根系酚 Root phenolics & 3.61 & 0.01 & $51.47^{* * *}$ & $9.75^{* *}$ & 0.00 & 0.01 & $33.56^{* * *}$ \\
\hline 茎叶水杨酸 Shoot salicylic acid & $49.52^{* * *}$ & 0.08 & 0.11 & $72.61^{* * *}$ & $80.09^{* * *}$ & $18.73^{* * *}$ & $95.11^{* * *}$ \\
\hline 茎叶茉莉酸 Shoot jasmonic acid & $156.85^{* * *}$ & 3.54 & $26.94^{* * *}$ & $38.62^{* * *}$ & $83.52^{* * *}$ & $64.95^{* * *}$ & $116.73^{* * *}$ \\
\hline
\end{tabular}

$* P<0.05, * * P<0.01, * * * P<0.001$. 

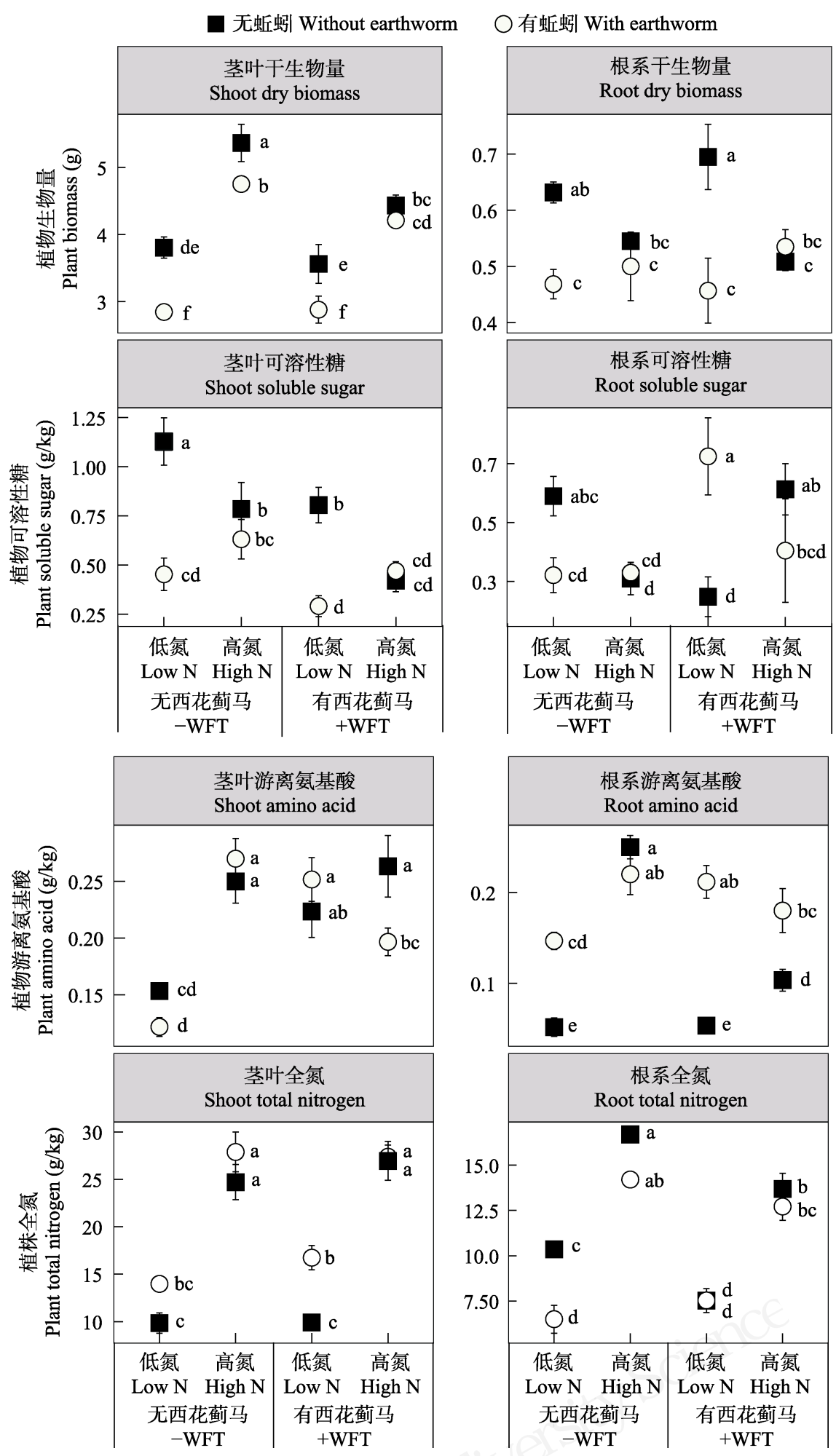

图 2 不同氮素条件下, 蚯蚓在西花蓟马(WFT)有无时对番茄茎叶干生物量、根系干生物量、茎十可溶性糖、根系可溶性糖、 茎叶游离氨基酸、根系游离氨基酸、茎叶全氮和根系全氮(平均值 \pm 标准差, $n=6$ )的影响。 $-W F T$ 和 $+W F T$ 分别表示无西 花蓟马和接种西花蓟马。不同小写字母表示各处理间差异显著(Fisher's LSD 检验, $\boldsymbol{P}<\boldsymbol{0 . 0 5}$ )。

Fig. 2 Effects of earthworm Metaphire guillelmi on the contents of tomato shoot dry biomass, root dry biomass, shoot soluble sugar, root soluble sugar, shoot amino acid, root amino acid, shoot total nitrogen and root total nitrogen in the absence or presence of western flower thrips under low and high $\mathrm{N}$ inputs (mean $\pm \mathrm{SD}, n=6$ ). In the figure, $-\mathrm{WFT}$ and $+\mathrm{WFT}$ indicate without and with western flower thrips, respectively. Means with different letters indicate significant difference among treatments (Fisher's LSD test, $P<0.05)$. Error bars are standard errors. 
量在蚯蚓作用下是未接种蝶蚓处理的6倍和3倍; 而 高氮下蝶蚓降低总酚含量, 对茎叶茉莉酸和水杨酸 含量无影响(图3)。西花蓟马数量的变化与氮素水平 和虾蚓关系密切, 在低氮时虾蚓使西花蓟马的数量 降低了 $58 \%$, 而高氮下无影响(图4)。在虾蚂存在时, 茎叶可溶性糖和茎叶全氮与西花蓟马数量显著正 相关, 茎叶茉莉酸和茎叶水杨酸含量与西花蓟马 数量呈显著负相关, 而无虾蚓时上述关系不显著 (图5)。

\section{3 讨论}

\section{1 蚯蚓对番茄生长的影响}

蚯蚓在低氮时降低了番茄茎叶干生物量、根系
干生物量和茎叶可溶性糖含量, 而高氮时对番茄营 养物质(可溶性糖、游离氨基酸和全氮)及生物量无 影响; 证实先前荟萃分析的结论，即虹蚓介导的植 物生长和养分分配受到土壤养分有效性的影响(van Groenigen et al, 2014)。蚯蚓活动影响着微生物活 性、群落结构及多样性(伍玉鹏等, 2013; Bertrand et al, 2015; Gong et al, 2018), 从而促进土壤有机氮矿 化，扩大土壤活性氮库(张卫信等，2007; Andriuzzi et al, 2016)。本研究中, 虾蚓活动增加了土壤微生物 生物量氮, 说明蝶蚓能够加速氮素周转, 这与番茄 茎叶全氮含量的增加相对应(Wurst，2013; van Groenigen et al, 2014)。蚯蚓降低了番茄的生物量和 茎叶可溶性糖, 其原因可能与威廉腔环蚓属于内层

无蚯蚓 Without earthworm

○有蚯蚓 With earthworm
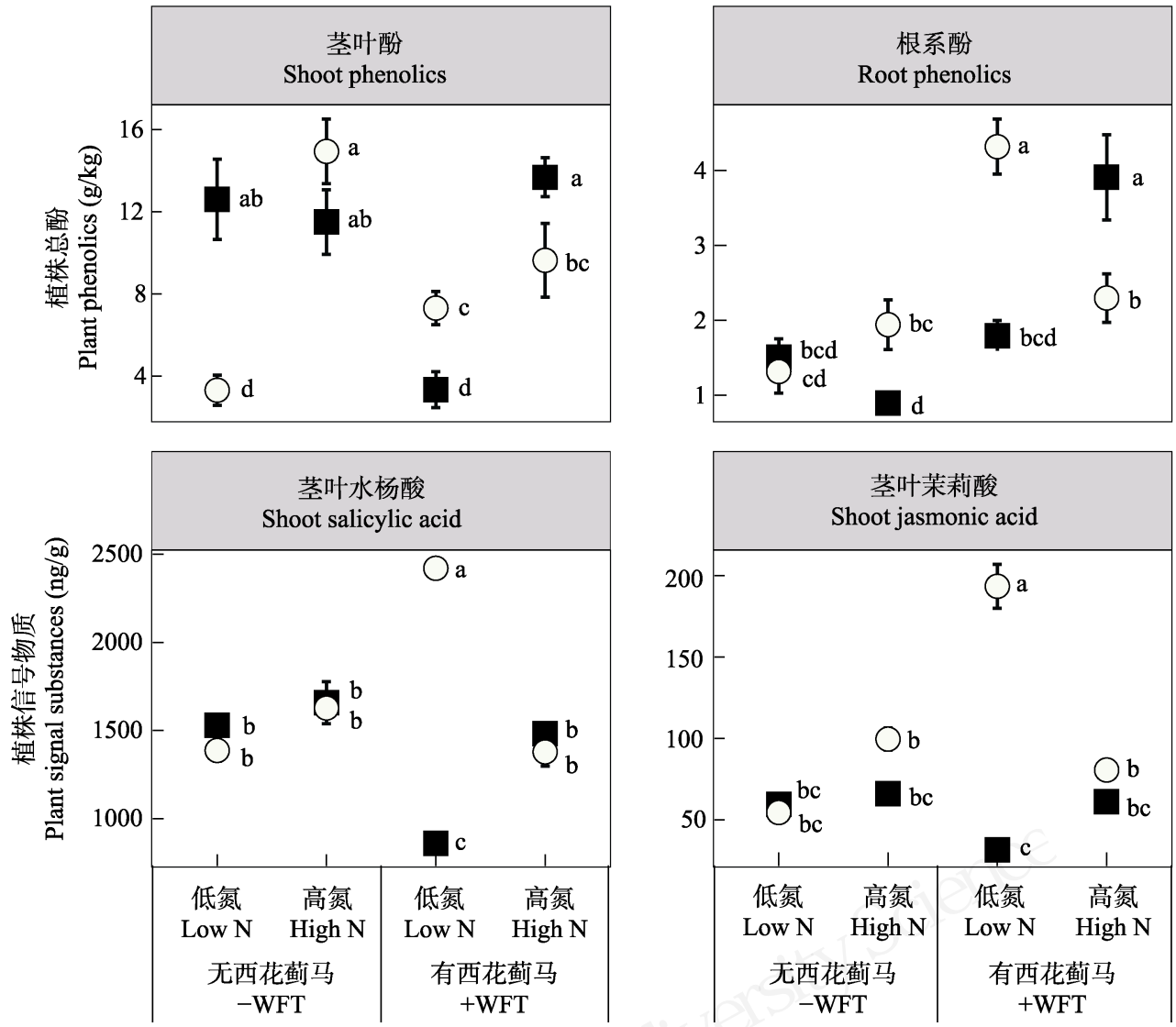

图3 不同氮素条件下, 蚳蚓在西花蓟马(WFT)有无时对番茄茎叶酚、根系酚、茎叶水杨酸和茎叶茉莉酸(平均值 \pm 标准差, $n=$ 6)的影响。-WFT 和+WFT 分别表示无西花蓟马和接种西花蓟马。不同小写字母表示各处理间差异显著(Fisher's LSD 检验, P 0.05)。

Fig. 3 Effects of earthworm Metaphire guillelmi on the contents of tomato shoot phenolics, root phenolics, shoot jasmonic acid and shoot salicylic acid in the absence or presence of western flower thrips (WFT) under low and high N inputs (mean \pm SD, $n=6$ ). In the figure, -WFT and +WFT indicate without and with western flower thrips, respectively. Means with different letters indicate significant difference among treatments (Fisher's LSD test, $P<0.05$ ). Error bars are standard errors. 


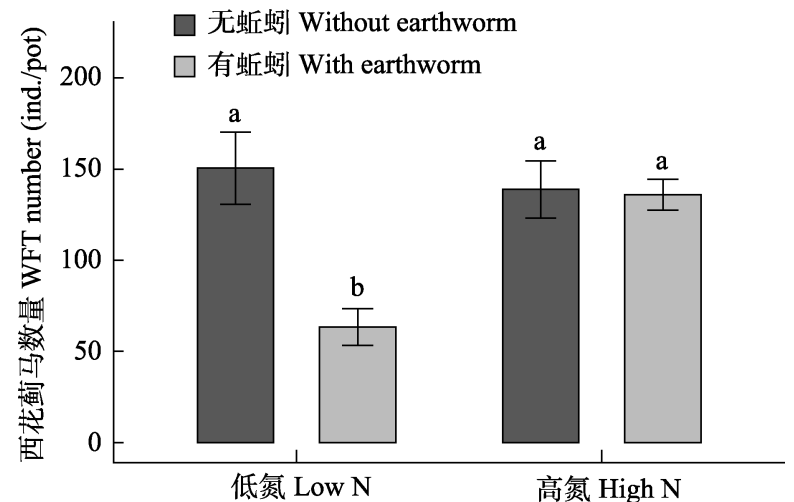

图4 蚯蚓和氮素水平对植食者西花蓟马(WFT)种群数量 (平均值 \pm 标准差, $n=6$ )的影响。不同小写字母表示各处理 间差异显著(Fisher's LSD 检验, $\boldsymbol{P}<\mathbf{0 . 0 5}$ )。

Fig. 4 Effects of earthworm and nitrogen level on the abundance of western flower thrips (WFT) (mean \pm SD, $n=6$ ). Means with different letters indicate significant difference among treatments (Fisher's LSD test, $P<0.05$ ). Error bars are standard errors.

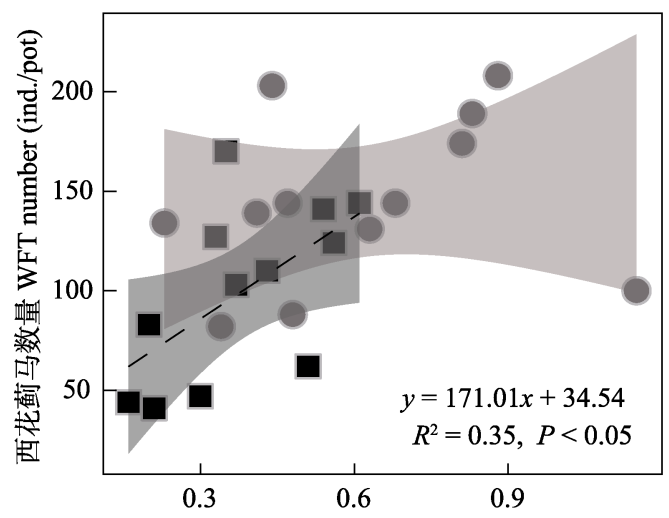

茎叶可溶性糖 Shoot soluble sugar $(\mathrm{g} / \mathrm{kg})$

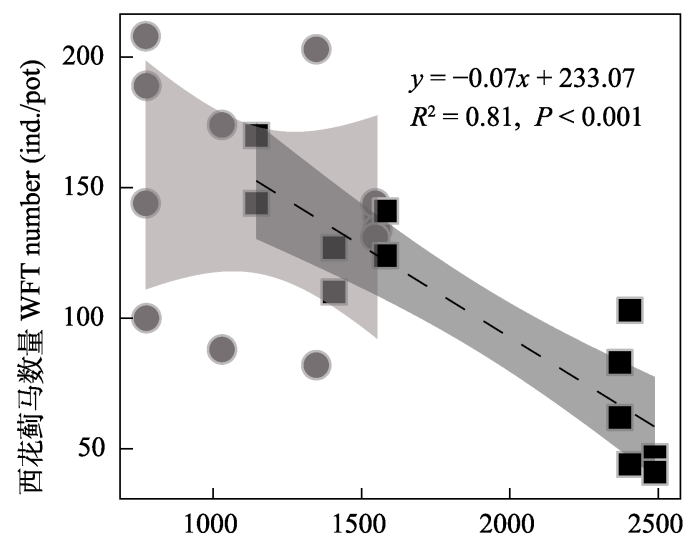

茎叶水杨酸 Shoot salicylic acid (ng/g)

无蚯蚓 Without earthworm
种，不仅对农田耕作和化肥施用等农事活动的抵抗 力强, 其本身对土壤结构和根系的生物扰动能力也 很强。尤其是在盆栽条件下，根系生长范围和蚳蚂 活动空间有限, 虾蚂对根系的破坏作用与地下部植 食者类似，从而对番茄的生长产生负面影响 (Kaplan et al, 2018)。西花薊马侵袭番茄后，可溶性 糖从地上部茎叶向地下部根系转移, 茎叶游离氨基 酸也因此增加，表明番茄在应对生物胁迫条件下， 其养分资源发生转移与再分配(Kempel et al, 2011)。 有文献也证明虾蚂活动能够造成根系破损, 特别是 未形成保护皮层的幼小细弱根及根尖，更容易受到 物理扰动的损害，同时虾蚓扰动也会破坏菌根菌丝 网络和有益真菌对植物的促进作用(Brown et al, 2004)。相比低氮水平, 高氮引起蜌蚂生物量降低的 程度更大, 尽管蝶蚂具有保持身体元素稳态的能力,

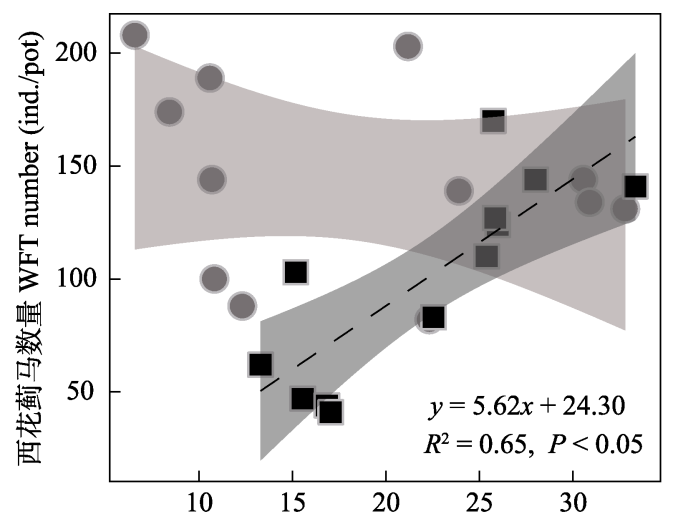

茎叶全氮 Shoot total nitrogen $(\mathrm{g} / \mathrm{kg})$

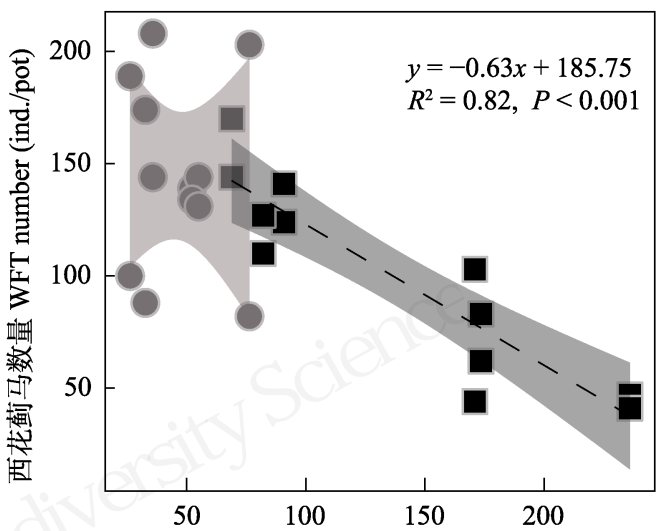

茎叶茉莉酸 Shoot jasmonic acid $(\mathrm{ng} / \mathrm{g})$

有虾蚓 With earthworm

图5 蚯蚓介导的番茄营养物质(茎叶可溶性糖和茎叶全氮)及信号物质(茎叶茉莉酸和茎叶水杨酸)与西花蓟马数量(WFT)的 关系

Fig. 5 Regressions between western flower thrips (WFT) abundance and contents of tomato shoot soluble sugar, shoot total nitrogen, shoot salicylic acid and shoot jasmonic acid. 
但高氮条件下蚯蚓摄取食物的元素不均衡会抑制 其生长(Zheng et al, 2018)。此外, 除了高氮水平使得 植物对蚟蚓介导的氮素矿化过程依赖性降低外, 氮 肥的施用伴随着 $\mathrm{pH}$ 的下降, 也会影响栖居于土壤 内的蚯蚓, 因此虹蚓在高氮条件下对植物生长的影 响较弱甚至被掩盖(Brown et al, 2004)。先前的综述 也发现, 在67项研究中有 $9 \%$ 的结果显示出蚟蚓降 低了植物生物量(Scheu, 2003), 除了蚯蚓取食和生 物扰动破坏了植物根系外, 有关蚯蚓影响根际微生 物群落的间接作用现在还缺乏研究。蝶蚛对植物生 长的影响可能取决于植物种类、土壤与植食者类型 的综合作用(Kaplan et al, 2018), 今后应该加强有关 蚯蚓影响植物生长的机制研究。

\section{2 蚯蚓对番茄抗虫性的影响}

植物对植食者的抗性受植物营养物质及化学 防御物质变化的影响(Kempel et al, 2009)。值得注意 的是, 本研究发现蝶蚓介导的生长和抗性之间并非 是此消彼长的关系, 蝶蚓可以同时促进植物营养积 累以及防御抗性。氮含量的增加会提高茎叶适口性, 增加西花蓟马的种群数量。而茉莉酸和水杨酸通过 细胞膜信号级联反应, 调控着抗性相关酶的活性及 抗性基因的表达, 进而刺激植物防御植食者(Jana et al, 2010; Puga-Freitas \& Blouin, 2015)。有研究证明 虹蚓会影响植物的信号途径(Lohmann et al, 2009)、 刺激植物叶片和皮下组织的防御化合物产生(Wurst et al, 2010); 如 Lohmann 等(2009)发现虹蚓会促进 叶片中次级防御化合物(如硫代葡萄糖苷)的形成来 降低蚜虫数量。植物生长和抗性所依赖的初级和次 级代谢途径是相互交织的, 大多数理论都强调资源 的最优分配及生长和防御之间存在此消彼长的权 衡(Wurst et al, 2010; Kempel et al, 2011)。虾蚓改善 土壤环境和调节土壤生物群落, 理论上可以影响植 食作用下资源在植物生长和防御之间的分配(张宁 等, 2012; Wurst, 2013)。与我们的推测相符, 蝶蚂影 响的方向和程度因土壤氮素水平而变化。在高氮条 件下, 蚯蚓对植物生长及抗性作用并不明显; 低氮 条件下, 蚯蚓促进植物对氮素的吸收, 同时也刺激 了番茄抗性化合物(茎叶酚)和抗性信号分子(茎叶 茉莉酸、茎叶水杨酸)的合成, 可能由于在抵抗西花 蓟马上植物消耗了大量的能量, 反而使其生长因蚯 蚓的存在而显示出被抑制的表象(Burghardt, 2016; Mundim et al, 2017)。碳氮平衡假说表明，土壤资源
有效性可以调节植物碳水化合物的储存, 改变植物 组织中碳基化感化合物的含量 (Jarzomski et al, 2000)。已有的证据表明低氮条件下植物会产生更多 代谢化合物, 如蛏蚓在氮充足条件下降低了车前草 (Plantaga lanceolate)中环烯醚萜类化合物的浓度, 抑制植食者(Wurst et al, 2004)。

随着蚳蚓一植物一植食者相互作用研究的不断 深入, 越来越多的研究结果表明, 蚯蚓介导的化学 防御物质的产生还受到植物基因型、土壤养分有效 性、植食者类型等生境因子的影响(Morris et al, 2006; Puga-Freitas \& Blouin, 2015)。因此, 深入揭示 蝶蚓对植物防御病原物的影响机制需要同时考虑 来自土壤管理和病虫害暴发等多方面的因素。本研 究仅涉及防御信号和总酚指标, 未测定抗性相关酶 活性及抗性基因等; 莱莉酸和水杨酸发挥其功能作 用并非简单的线性途径，不同途径构成复杂的信号 转导网络, 共同调控植物的防御抗性(Derksen et al, 2013)。蚯蚓对植物生长及抗性的影响机制极其复杂, 其影响程度和方向仍难以得到一致的结论。虾蚓介 导的植物抵抗植食者的能力还可能取决于根际微 生物组(Wurst \& Forstreuter, 2010; Paudel et al, 2016), 如土壤中的一些有益微生物能促进植物防 御物质的合成(Eisenhauer et al, 2010; 李欢等, 2011; Pineda et al, 2015)。今后应更重视虹蚓介导的上行 效应(Bottom-up effects), 有关研究将有助于发展土 壤生态管理措施, 促进土壤动物资源的调控和利用, 发挥土壤生物在植物防御病虫害方面的作用。

\section{结论}

植物的抗虫性高低与植物体营养物质及防御 物质含量有关。本研究发现蚯蚓介导的植物防御化 合物以及抗逆信号可以加强其对地上部虫害的抗 性。植物生长和抗性之间的关系受土壤养分水平的 影响, 蚯蚓仅在低氮时提高了植物的抗虫性。可见, 养分富集条件下植物与土壤动物的互利关系受到 破坏。今后应基于野外自然条件或较长时空尺度、 结合土壤生物群落的系统分析及植物防御代谢组 学研究, 剖析蛏蚓介导的植物生长及抗性的关系及 调控机制。

致谢: 感谢南京农业大学资源与环境科学学院王 帅、罗琌、周星、王震威和靳楠同学的帮助。 


\section{参考文献}

Ainsworth EA, Gillespie KM (2007) Estimation of total phenolic content and other oxidation substrates in plant tissues using Folin-Ciocalteu reagent. Nature Protocols, 2, 875-877.

Andriuzzi WS, Schmidt O, Brussaard L, Faber JH, Bolger T (2016) Earthworm functional traits and interspecific interactions affect plant nitrogen acquisition and primary production. Applied Soil Ecology, 104, 148-156.

Aurélien R, Michael G, Sergio R, Sanders IR (2013) Identity and combinations of arbuscular mycorrhizal fungal isolates influence plant resistance and insect preference. Ecological Entomology, 38, 330-338.

Bardgett RD, van der Putten WH (2014) Belowground biodiversity and ecosystem functioning. Nature, 515, 505-511.

Bender SF, Wagg C, van der Heijden MGA (2016) An underground revolution: Biodiversity and soil ecological engineering for agricultural sustainability. Trends in Ecology and Evolution, 31, 440-452.

Bertrand M, Barot S, Blouin M, Whalen J, de Oliveira T, Roger-Estrade J (2015) Earthworm services for cropping systems: A review. Agronomy for Sustainable Development, 35, 553-567.

Blouin M, Zuily-Fodil Y, Pham-Thi AT, Laffray D, Reversat G, Pando A, Tondoh JE, Lavelle P (2005) Belowground organism activities affect plant aboveground phenotype, inducing plant tolerance to parasites. Ecology Letters, 8, 202-208.

Bogaert F, Chesnais Q, Catterou M, Rambaud C, Geraldine D, Ameline A (2017) How the use of nitrogen fertiliser may switch plant suitability for aphids: The case of Miscanthus, a promising biomass crop and the aphid pest Rhopalosiphum maidis. Pest Management Science, 73, 1648-1654.

Bommarco R, Kleijn D, Potts SG (2013) Ecological intensification: Harnessing ecosystem services for food security. Trends in Ecology and Evolution, 28, 230-238.

Brown GG, Edwards CA, Brussaard L (2004) How earthworms affect plant growth: Burrowing into the mechanisms. Earthworm Ecology, 2, 13-49.

Burghardt KT (2016) Nutrient supply alters goldenrod's induced response to herbivory. Functional Ecology, 30, 1769-1778.

Caarls L, Pieterse CM, van Wees S (2015) How salicylic acid takes transcriptional control over jasmonic acid signaling. Frontiers in Plant Science, 6, 170.

Dalby P, Baker G, Smith S (1996) "Filter paper method" to remove soil from earthworm intestines and to standardise the water content of earthworm tissue. Soil Biology and Biochemistry, 28, 685-687.

Derksen H, Rampitsch C, Daayf F (2013) Signaling cross-talk in plant disease resistance. Plant Science, 207, 79-87.

Eisenhauer N, Hoersch V, Moeser J, Scheu S (2010) Synergistic effects of microbial and animal decomposers on plant and herbivore performance. Basic and Applied Ecology, 11, 23-34.

Fu SL (2007) A review and perspective on soil biodiversity research. Biodiversity Science, 15, 109-115. (in Chinese with English abstract) [傅声雷 (2007) 土壤生物多样性的 研究概况与发展趋势. 生物多样性, 15, 109-115.]

Gong X, Jiang YY, Zheng Y, Chen XY, Li HX, Hu F, Liu MQ, Scheu $S$ (2018) Earthworms differentially modify the microbiome of arable soils varying in residue management. Soil Biology and Biochemistry, 121, 120-129.

Hettenhausen C, Baldwin IT, Wu J (2013) Nicotiana attenuata MPK4 suppresses a novel jasmonic acid (JA) signalingindependent defense pathway against the specialist insect Manduca sexta, but is not required for the resistance to the generalist Spodoptera littoralis. New Phytologist, 199, 787-799.

Jana U, Barot S, Blouin M, Lavelle P, Laffray D, Repellin A (2010) Earthworms influence the production of above- and belowground biomass and the expression of genes involved in cell proliferation and stress responses in Arabidopsis thaliana. Soil Biology and Biochemistry, 42, 244-252.

Jarzomski CM, Stamp NE, Bowers MD (2000) Effects of plant phenology, nutrients and herbivory on growth and defensive chemistry of plantain, Plantago lanceolata. Oikos, 88, 371-379.

Kaplan I, Pineda A, Bezemer M (2018) Application and theory of plant-soil feedbacks on aboveground herbivores. In: Aboveground-Belowground Community Ecology (eds Ohgushi T, Wurst S, Johnson SN), pp. 319-343. Springer International Publishing, West Lafayette.

Kempel A, Brandl R, Schadler M (2009) Symbiotic soil microorganisms as players in aboveground plant-herbivore interactions - the role of rhizobia. Oikos, 118, 634-640.

Kempel A, Schaedler M, Chrobock T, Fischer M, van Kleunen M (2011) Tradeoffs associated with constitutive and induced plant resistance against herbivory. Proceedings of the National Academy of Sciences, USA, 108, 5685-5689.

Korres NE, Norsworthy JK, Tehranchian P, Gitsopoulos TK, Loka DA, Oosterhuis DM, Gealy DR, Moss SR, Burgos NR, Miller MR, Palhano M (2016) Cultivars to face climate change effects on crops and weeds: A review. Agronomy for Sustainable Development, 36, 1-22.

Larbat R, Olsen KM, Slimestad R, Lovdal T, Benard C, Verheul M, Bourgaud F, Robin C, Lillo C (2012) Influence of repeated short-term nitrogen limitations on leaf phenolics metabolism in tomato. Phytochemistry, 77, 119-128.

Li H, Li XL, Zhang JL, Gai JP, Wang C, Xiang D (2011) Interaction between earthworm and AM fungi and their effects on plant performance. Acta Pedologica Sinica, 48, 847-855. (in Chinese with English abstract) [李欢, 李晓林, 张俊伶, 盖京苹, 王冲, 向丹 (2011) 蚟蚓与丛枝菌根真 菌的相互作用及其对植物的影响. 土壤学报, 48, 847-855.] 
Lohmann M, Scheu S, Muller C (2009) Decomposers and root feeders interactively affect plant defence in Sinapis alba. Oecologia, 160, 289-298.

Loranger-Merciris G, Cabidoche YM, Delone B, Queneherve P, Ozier-Lafontaine H (2012) How earthworm activities affect banana plant response to nematodes parasitism. Applied Soil Ecology, 52, 1-8.

Lu RK (2000) Analysis Method of Soil Agricultural Chemistry. China Agricultural Science and Technology Press, Beijing. (in Chinese) [鲁如坤 (2000) 土壤农业化学分析方法. 中 国农业科技出版社, 北京.]

Lü YB, Zhang ZJ, Wu QJ, Du YZ, Zhang HR, Yu Y, Wang ED, Wang MH, Wang MQ, Tong XL, Lü LH, Tan XQ, Fu WD (2011) Research progress of the monitoring, forecast and sustainable management of invasive alien pest Frankliniella occidentalis in China. Chinese Journal of Applied Entomology, 48, 488-496. (in Chinese with English abstract) [吕要斌, 张治军, 吴青君, 杜予州, 张宏瑞, 于 毅, 王恩东, 王鸣华, 王满困, 童晓立, 吕利华, 谭新球, 付卫东 (2011) 外来入侵害虫西花蓟马防控技术研究与 示范. 应用昆虫学报, 48, 488-496.]

Morris WF, Traw MB, Bergelson J (2006) On testing for a tradeoff between constitutive and induced resistance. Oikos, 112, 102-110.

Mundim FM, Alborn HT, Vieira-Neto EHM, Bruna EM (2017) A whole-plant perspective reveals unexpected impacts of above- and belowground herbivores on plant growth and defense. Ecology, 98, 70-78.

Pan KW, Zhang L, Shao YH, Fu SL (2016) Thematic monitoring network of soil fauna diversity in China: Exploring the mystery of soil. Biodiversity Science, 24, 1234-1239. (in Chinese with English abstract) [潘开文, 张 林, 邵元虎, 傅声雷 (2016) 中国土壤动物多样性监测: 探知土壤中的奥秘. 生物多样性, 24, 1234-1239.]

Paudel S, Longcore T, MacDonald B, Mccormick MK, Szlavecz K, Wilson GWT, Loss SR (2016) Belowground interactions with aboveground consequences: Invasive earthworms and arbuscular mycorrhizal fungi. Ecology, 97, 605-614.

Pineda A, Soler R, Pozo MJ, Rasmann S, Turlings TCJ (2015) Above-belowground interactions involving plants, microbes and insects. Frontiers in Plant Science, 6, 318.

Poveda K, Steffan-Dewenter I, Scheu S, Tscharntke T (2005) Effects of decomposers and herbivores on plant performance and aboveground plant-insect interactions. Oikos, 108, 503-510.

Puga-Freitas R, Blouin M (2015) A review of the effects of soil organisms on plant hormone signalling pathways. Environmental and Experimental Botany, 114, 104-116.

Schädler M, Ballhorn DJ (2017) Beneficial soil microbiota as mediators of the plant defensive phenotype and aboveground plant-herbivore interactions. In: Progress in Botany (eds Cánovas FM, Lüttge U, Matyssek R), pp. 305-343.
Springer International Publishing, Berlin.

Scheu S (2003) Effects of earthworms on plant growth: Patterns and perspectives. Pedobiologia, 47, 846-856.

Shekoofa A, Emam Y (2008) Effects of nitrogen fertilization and plant growth regulators (PGRs) on yield of wheat (Triticum aestivum L.) cv. Shiraz. Journal of Agricultural Science and Technology, 10, 101-108.

Staley JT, Stewartjones A, Pope TW, Wright DJ, Leather SR, Hadley P, Rossiter JT, van Emden HF, Poppy GM (2010) Varying responses of insect herbivores to altered plant chemistry under organic and conventional treatments. Proceedings of the Royal Society B: Biological Sciences, 277, 779-786.

Tao L, Ahmad A, Roode JC, Hunter MD (2016) Arbuscular mycorrhizal fungi affect plant tolerance and chemical defences to herbivory through different mechanisms. Journal of Ecology, 104, 561-571.

Tsiafouli MA, Thebault E, Sgardelis SP, Deruiter PC, van der Putten WH, Birkhofer RD, Hemerik L, de Vries FT, Bargett RD, Brady MV, Bjornlund L, Jorgensen HB, Christensen S, Hertefeldt TD, Hotes S, Hol WHG, Frouz J, Liiri M, Mortimer SR, Setala H, Tzanopoulos J, Uteseny K, Pizl V, Stary J, Wolters V, Hedlund K (2015) Intensive agriculture reduces soil biodiversity across Europe. Global Change Biology, 21, 973-985.

van Groenigen JW, Lubbers IM, Vos HM, Brown GG, Deyn GB, van Groenigen KJ (2014) Earthworms increase plant production: A meta-analysis. Scientific Reports, 4, 6365.

Wang SJ, Ruan HH (2008) Feedback mechanisms of soil biota to aboveground biology in terrestrial ecosystems. Biodiversity Science, 16, 407-416. (in Chinese with English abstract) [王邵军, 阮宏华 (2008) 土壤生物对地上生物 的反馈作用及其机制. 生物多样性, 16, 407-416.]

Wu JH, Song CY, Chen JK (2007) Effect of microbivorous nematodes on plant growth and soil nutrient cycling: A review. Biodiversity Science, 15, 124-133. (in Chinese with English abstract) [吴纪华, 宋慈玉, 陈家宽 (2007) 食微 线虫对植物生长及土壤养分循环的影响. 生物多样性, 15, 124-133.]

Wu YP, Lü LY, Bi YM, Zhang Y, Sun ZJ (2013) Effects of earthworm inoculation on saline-alkali soil nutrient, soil organisms and plant cultivation. Journal of China Agricultural University, 18, 45-51. (in Chinese with English abstract) [伍玉鹏, 吕丽媛, 毕艳孟, 张一, 孙振钧 (2013) 接种蝶蚓对盐碱土养分、土壤生物及植被的影响. 中国农 业大学学报, 18, 45-51.]

Wurst S (2013) Plant-mediated links between detritivores and aboveground herbivores. Frontiers in Plant Science, 4, 380.

Wurst S, Dugassa-Gobena D, Langel R, Bonkowski M, Scheu $S$ (2004) Combined effects of earthworms and vesicular-arbuscular mycorrhizas on plant and aphid performance. New Phytologist, 163, 169-176.

Wurst S, Wagenaar R, Biere A, van der Putten WH (2010) 
Microorganisms and nematodes increase levels of secondary metabolites in roots and root exudates of Plantago lanceolata. Plant and Soil, 329, 117-126.

Wurst S, Forstreuter M (2010) Colonization of Tanacetum vulgare by aphids is reduced by earthworms. Entomologia Experimentalis et Applicata, 137, 86-92.

Xiao ZG, Wang X, Koricheva J, Kergunteuil A, Le Bayon R, Liu MQ, Hu F, Rasmann S (2018) Earthworms affect plant growth and resistance against herbivores: A meta-analysis. Functional Ecology, 32, 150-160.

Xu W, Ma ZY, Jing X, He JS (2016) Biodiversity and ecosystem multifunctionality: Advances and perspectives. Biodiversity Science, 24, 55-71. (in Chinese with English abstract) [徐炜, 马志远, 井新, 贺金生 (2016) 生物多样 性与生态系统多功能性: 进展与展望. 生物多样性, 24, 55-71.]

Zhang N, Liao Y, Sun FL, Wang C, Sun ZJ (2012) Earthworm population characteristics in soils different in land use and their relationships with biological fertility of the soils. Acta Pedologica Sinica, 49, 364-372. (in Chinese with English abstract) [张宁, 廖燕, 孙福来, 王冲, 孙振钧 (2012) 不
同土地利用方式下的蛏蚓种群特征及其与土壤生物肥力 的关系. 土壤学报, 49, 364-372.]

Zhang SJ, Chao Y, Zhang CL, Cheng J, Li J, Ma N (2010) Earthworms enhanced winter oilseed rape (Brassica napus L.) growth and nitrogen uptake. Agriculture Ecosystems and Environment, 139, 463-468.

Zhang WX, Chen DM, Zhao CC (2007) Functions of earthworm in ecosystem. Biodiversity Science, 15, 142-153. (in Chinese with English abstract) [张卫信, 陈迪马, 赵灿 灿 (2007) 蚯蚓在生态系统中的作用. 生物多样性, 15, 142-153.]

Zheng Y, Wang S, Bonkowski M, Chen XY, Griffiths B, Hu F, Liu MQ (2018) Litter chemistry influences earthworm effects on soil carbon loss and microbial carbon acquisition. Soil Biology and Biochemistry, 123, 105-114.

Züst T, Agrawal AA (2017) Trade-offs between plant growth and defense against insect herbivory: An emerging mechanistic synthesis. Annual Review of Plant Biology, 68, 513-534. 